\title{
MộT SỐ NHÂN TỐ ẢNH HƯởNG ĐẾN NỖ LỰC HỌC TẬP CỦA SINH VIÊN TRƯỜNG ĐẠI HỌC CÔNG NGHIẸP TPHCM
}

\author{
NGÔ NGỌC HUNG, NGUYỄN THI THU TRANG, TRẦ ANH DŨNG \\ Khoa Khoa học Cơ bản, trường Đại học Công nghiệp Thành phố Hồ Chí Minh \\ ngongochung@iuh.edu.vn
}

\begin{abstract}
Tóm tắt. Nghiên cứu này nhằm xác định ảnh hưởng của các hoạt động giảng dạy và hỗ trợ sinh viên (HĐGD\&HT) của trường Đại học Công nghiệp TPHCM (ĐHCN TPHCM) đối với nỗ lực học tập của sinh viên (NLHT). Dữ liệu được thu thập qua hình thức khảo sát bằng phiếu câu hỏi với sự tham gia của 500 sinh viên. Các HĐGD\&HT được sắp xếp vào 10 nhân tố, đóng vai trò như biến độc lập trong mô hình nghiên cứu. Kết quả nghiên cứu cho thấy (i) tất cả các nhân tố đều có mối tương quan đồng biến với nỗ lực học tập của sinh viên; (ii) xét tổng thể, các nhân tố có ảnh hưởng tích cực đến NLHT, tuy nhiên mức ảnh hưởng chưa cao; (iii) 5/10 nhân tố bao gồm Chiến lược học tập; Tương tác giữa giảng viên và sinh viên; Thách thức thi cử; Hợp tác trong học tập; Chất lượng tương tác (sắp xếp theo thứ tự giảm dần) có ảnh hưởng tích cực đến NLHT; (iv) các nhân tố Tư duy bậc cao; Học tập thông qua tích hợp và chiêm nghiệm; Lập luận định lượng; Hoạt động giảng dạy hiệu quả; Sự hỗ trợ của nhà trường không có ảnh hưởng đối với NLHT. Để thúc đẩy sinh viên nỗ lực học tập, ĐHCN TPHCM cần phải tăng cường các hoạt động phát triển tư duy bậc cao, học tập thông qua tích hợp và chiêm nghiệm, lập luận định lượng trong chương trình học và kiểm tra, đánh giá; tạo điều kiện để sinh viên tương tác nhiều hơn với giảng viên; tăng độ khó và đa dạng hóa hình thức của các kỳ thi.
\end{abstract}

Từ khóa. Nỗ lực học tập, hoạt động giảng dạy và hỗ trợ sinh viên, phát triển tư duy bậc cao, tương tác giữa sinh viên và giảng viên, học tập tích hợp và chiêm nghiệm.

\section{FACTORS INFLUENCING STUDENTS'ACADEMIC EFFORT OF INDUSTRIAL UNIVERSITY OF HO CHI MINH CITY}

\begin{abstract}
This study aimed at investigating the influence of teaching and supportive practices of Industrial University of Ho Chi Minh city (IUH) on student academic effort. A survey with the participation of 500 students was employed to collect data. The teaching and supportive practices were grouped into 10 factors, acting as independent variables in the research model. The research findings showed (i) all factors had positive correlations with student academic effort; (ii) 10 factors as a whole had positive effect on student academic effort but the effect level was not high; (iii) 5/10 factors, including Learning strategies; Student - lecturer interaction; Examination challenge; Collaborative learning; and Quality of interaction (in descending order) significantly and positively affected student academic effort; (iv) factors such as Higherorder thinking; Integrative and reflective learning; Quantitative reasoning; Effective teaching practices; and Institutional support had no influence on student academic effort. To promote students' academic effort, IUH should enhance the development of higher-order thinking, integrative and reflective learning and quantitative reasoning in its academic programmes and learning assessment; create favourable conditions for more student-lecturer interaction; increase the degree of difficulty of examinations and diversify types of examinations.
\end{abstract}

Keywords. Student academic effort, teaching and supportive practices, higher-order thinking, integrative and reflective learning, student-lecturer interaction.

\section{1.ĐẠT VẤN ĐỀ}

Cần cù, nỗ lực trong học tập là phẩm chất tốt đẹp của nhiều thế hệ sinh viên Việt Nam, nhưng hiện nay phẩm chất này đang bị mai một dần trong một bộ phận sinh viên. Một số nghiên cứu $[1,2]$ và một vài bài báo trên phương tiện thông tin đại chúng đã phản ánh hiện tượng một số sinh viên ít chuyên cần trong học tập. Họ dành nhiều thời gian để giải trí, tương tác trên mạng xã hội hay đi làm thêm hơn là dành thời gian cho học tập. Họ thường xuyên không chuẩn bị bài khi đến lớp, ít tập trung trong giờ học. Sự lơ là trong học 
tập của sinh viên đã dẫn đến một số hậu quả [2]. Đầu tiên, nó sẽ làm giảm chất lượng đào tạo đại học do có một số lượng lớn sinh viên không thể hoàn tất chương trình học đúng thời hạn hay bị cho thôi học. Báo chí đã phản ánh khá nhiều về tỷ lệ sinh viên bị buộc thôi học tăng đột biến trong những năm gần đây [2]. Một số sinh viên khác hoàn thành được chương trình học nhưng không tích lũy đủ kiến thức và kỹ năng để tham gia vào thị trường lao động. Theo thống kê mới nhất của Bộ Lao động, Thương binh và Xã hội, trong quý 2/2018, có hơn 126.900 sinh viên tốt nghiệp đã không thể tìm được việc làm sau khi tốt nghiệp. Việc nhiều sinh viên thất nghiệp cũng có những ảnh hưởng nhất định đến các trường đại học Việt Nam. Nó làm giảm sức hấp dẫn của giáo dục đại học đối với phụ huynh và học sinh. Học sinh sẽ cân nhắc xem có nên thi vào đại học hay không. Điều này có thể dẫn đến số lượng sinh viên đăng ký tuyển sinh vào đại học giảm. Một số sinh viên gia đình có điều kiện sẽ nghĩ đến việc học đại học ở nước ngoài thay vì học trong nước. Các yếu tố trên sẽ làm cho cuộc cạnh tranh trong tuyển sinh giữa các trường đại học ngày càng khốc liệt hơn.

Thực trạng trên cho thấy đã đến lúc các nhà quản lý và nghiên cứu giáo dục đại học ở Việt Nam cần quan tâm hơn đến nỗ lực học tập của sinh viên (NLHT), và quan trọng hơn, những biện pháp có thể tăng cường NLHT. Nghiên cứu này được thực hiện nhằm tìm hiểu ảnh hưởng của các hoạt động giảng dạy và hỗ trợ sinh viên (HĐGD\&HT) của trường Đại học Công nghiệp TPHCM (ĐHCN TPHCM) đối với NLHT. Nghiên cứu sẽ kiểm tra mức độ ảnh hưởng chung của các HĐGD\&HT đối với NLHT cũng như xác định mức độ ảnh hưởng của từng HĐGD\&HT đổi với NLHT. Qua đó, nghiên cứu sẽ xác định những HĐGD\&HT nào có tác động tích cực nhất đến NLHT, những HĐGD\&HT nào chưa có tác động như mong muốn đối với NLHT và nguyên nhân vì sao. Từ kết quả nghiên cứu này, nhóm nghiên cứu sẽ đưa ra một số khuyến nghị để ĐHCN TPHCM có thể khuyến khích, thúc đẩy sinh viên nỗ lực học tập nhằm nâng cao chất lượng đào tạo của mình. Về mặt lý luận, theo hiểu biết của nhóm nghiên cứu, tính đến nay chưa có nghiên cứu nào ở Việt Nam kiểm tra mối quan hệ giữa HĐGD\&HT của nhà trường và NLHT. Trên thế giới, mặc dù có nhiều nghiên cứu về NLHT và HĐGD\&HT của nhà trường nhưng trong các nghiên cứu này, NLHT và HĐGD\&HT của nhà trường được xem như là 2 khía cạnh của khái niệm Student Engagement và chủ yếu các nhà nghiên cứu kiểm tra ảnh hưởng của Student Engagement đối với kết quả học tập và rèn luyện của sinh viên. Từ những nguyên nhân trên, nghiên cứu này sẽ có những đóng góp nhất định vào hệ thống tri thức của thế giới về NLHT và các HĐGD\&HT của nhà trường. Nghiên cứu cũng đặt nền móng cho các nghiên cứu chuyên sâu hơn về NLHT và HĐGD\&HT của nhà trường ở Việt Nam.

\section{TỔNG QUAN TÀI LIÊUU}

\subsection{Nỗ lực học tập của sinh viên (NLHT)}

Nỗ lực học tập của sinh viên (NLHT) luôn được xem là một trong những nhân tố có ảnh hưởng tích cực đến kết quả học tập, kết quả rèn luyện và phát triển nhân cách của sinh viên trong thời gian học đại học. Theo Kuh (2009), NLHT là sự đầu tư về thời gian, là chất lượng của những nỗ lực mà sinh viên dành cho việc học tập cũng như tham gia vào các hoạt động có liên quan đến học tập. Chất lượng của nỗ lực được định nghĩa như sự đầu tư sức lực và trí tuệ của sinh viên để hoàn thành các nhiệm vụ học tập ở mức cao nhất có thể [3]. Trong một số nghiên cứu ở Mỹ [4, 5], các chỉ báo của NLHT bao gồm thời gian sinh viên dành cho việc học tập; sự tự giác chuẩn bị bài, hoàn tất bài tập đầy đủ trước khi đến lớp; sự cố gắng để nắm vững các nội dung khó trong bài, để hoàn thiện các nhiệm vụ học tập ở mức tốt nhất có thể, để đáp ứng được ở mức cao nhất có thể các yêu cầu của giảng viên, của chương trình học. NLHT còn thể hiện qua việc chủ động, tích cực tìm kiếm các cơ hội học tập như tương tác với bạn học, với giảng viên, sử dụng hiệu quả các nguồn tài nguyên (thư viện, phòng máy...), các hoạt động tư vấn, hồ trợ do nhà trường cung cấp. Chính sự chuyên cần, tự giác trong học tập này sẽ giúp sinh viên nắm vững các kiến thức và kỹ năng cần thiết, tận dụng tốt được các cơ hội học tập, khám phá và phát huy được năng lực của bản thân để thành công trong công việc và cuộc sống sau này [3]. NLHT có thể chịu tác động của một số yếu tố chủ quan như động cơ học tập và mục đích học tập. Động cơ học tập là động lực thúc đẩ sinh viên thực hiện các nhiệm vụ học tập [6]. Mục đích học tập định hướng cho NLHT của sinh viên, giúp sinh viên duy trì sự kiên trì̀, bền bỉ trong học tập [7]. Khi đặt ra mục đích học tập càng cao, sinh viên càng nỗ lực nhiều trong học tập [8].

\subsection{Hoạt động giảng dạy, hỗ trọ sinh viên (HĐGD\&HT) của nhà trường}

Sinh viên đóng vai trò chủ đạo trong NLHT thế nhưng thực tế cho thấy không có nhiều sinh viên tự giác nỗ lực trong học tập. Một nghiên cứu về phong cách học của sinh viên được tiến hành tại trường Đại học Quốc Gia Hà Nội năm 2008 cho thấy mặc dù có đến 82,5\% sinh viên công nhận ảnh hưởng quyết định của 
TRƯỜNG ĐẠI HỌC CÔNG NGHIỆP TPHCM

NLHT đối với kết quả học tập nhưng chỉ có khoảng 50,3\% sinh viên cho biết họ bỏ ra vài giờ mỗi ngày để tự học và chỉ có $54,2 \%$ sinh viên ra sức học tập để hoàn thành mục tiêu học tập của mình [11]. Kết quả nghiên cứu này chỉ ra rằng để sinh viên nỗ lực học tập cần phải có tác động từ phía nhà trường. Bằng các nguồn lực (cơ sở vật chất, đội ngũ giảng viên, cán bộ ...), các chương trình học, các dịch vụ hỗ trợ, nhà trường có thể tạo ra nhiều cơ hội cho sinh viên tham gia vào các hoạt động học tập [12]. Theo các nhà nghiên cứu Mỹ, các biện pháp mà nhà trường có thể sử dụng để nâng cao nỗ lực học tập của sinh viên bao gồm:

- Đặt ra cho sinh viên các thách thức học thuật. Thách thức học thuật được tạo dựng qua việc đặt các tiêu chuẩn và kỳ vọng cao nhưng không vượt quá khả năng của sinh viên. Những tiêu chuẩn này sẽ buộc sinh viên phải nỗ lực tối đa mới có thể đạt được kết quả học tập tốt [8]. Thách thức học thuật còn được thực hiện thông qua sự chú trọng của chương trình học, kiểm tra, đánh giá đến việc hình thành và phát triển ở sinh viên các kỹ năng tổng hợp, phân tích, đánh giá thông tin, kỹ năng ứng dụng kiến thức vào thực tế, kỹ năng tích hợp thông tin và chiêm nghiệm các trải nghiệm học tập, khả năng hiểu và sử dụng các thông tin dạng số của sinh viên để đưa ra kết luận, để nhận định và đánh giá các lập luận hay vấn đề [11]. Khi thực hiện những hoạt động này, sinh viên sẽ phải tư duy nhiều hơn, phải dành nhiều thời gian hơn cho học tập. Những hoạt động này cũng sẽ khơi gợi sự hứng thú của sinh viên đối với học tập. Áp lực từ phía nhà trường cộng với áp lực học tập tự đặt cho bản thân sẽ thúc đẩy sinh viên cố gắng vượt qua giới hạn của mình để nâng cao kiến thức hoặc chuyên tâm lâu dài với việc học tập [12]. Tuy vậy, để đạt được kết quả như mong muốn, áp lực học tập cần phải đi đôi với sự hỗ trợ sinh viên trong học tập. Sự hỗ trợ thể hiện qua các hoạt động định hướng, tư vấn, các trung tâm bồi dưỡng các kỹ năng học thuật cần thiết nhưng còn thiếu cho sinh viên cũng như giúp đỡ cho các sinh viên gặp khó khăn trong học tập [10, 13].

- Hoạt động giảng dạy hiệu quả của giảng viên. Những hoạt động này bao gồm việc định hướng môn học rõ ràng cho sinh viên, trình bày bài giảng logic, sử dụng các ví dụ, minh họa để giảng giải bài học, cũng như đưa ra các phản hồi chi tiết, kịp thời, thường xuyên về các bài tập, bài kiểm tra của sinh viên [11]. Điều này sẽ giúp sinh viên tiếp thu kiến thức hiệu quả hơn, qua đó, có tác động tích cực đến kết quả học tập của sinh viên. Bên cạnh đó, nhà trường cũng cần tạo điều kiện để sinh viên có thể tương tác với giảng viên, ví dụ như sinh viên có thể tiếp cận và tham khảo ý kiến của giảng viên về các vấn đề liên quan đến học tập và hướng nghiệp hay tham gia cùng giảng viên trong các hoạt động nghiên cứu và các hoạt động ngoại khóa [11]. Sự tư vấn, động viên từ giảng viên sẽ giúp sinh viên mở mang kiến thức và phát triển kỹ năng, xác định được mục tiêu học tập, hứng thú trong học tập, nhờ đó, sinh viên sẽ nỗ lực hơn trong học tập [10];

- Tạo điều kiện để sinh viên có thể hợp tác với các bạn học trong các hoạt động học tập khác nhau (giải thích bài học, chuẩn bị các bài thi, làm việc nhóm trong các đồ án hay tiểu luận). Khi làm việc nhóm, sinh viên sẽ phải bỏ nhiều thời gian, công sức hơn cho việc học tập. Bên cạnh đó, sự hợp tác này sẽ giúp sinh viên củng cố kiến thức, phát triển các kỹ năng (làm việc nhóm, giao tiếp, giải quyết vấn đề ...), tạo sự gắn kết giữa các sinh viên với nhau [11].

- Hỗ trợ sinh viên thông qua các chính sách, các trung tâm và dịch vụ giúp đỡ sinh viên trong cả học tập và cuộc sống, đồng thời tạo ra một bầu không khí thân thiện giữa sinh viên với bạn học, với giảng viên, cố vấn học tập, cán bộ, nhân viên. Sự chăm sóc và giúp đỡ sinh viên đạt thành quả trong học tập và phát triển nhân cách luôn được xem là một trong những nhiệm vụ quan trọng hàng đầu của các trường đại học phương Tây [14]. Một môi trường hỗ trợ, thân thiện sẽ tạo cho sinh viên sự hài lòng với nhà trường, giúp sinh viên hòa nhập, gẳn bó với nhà trường và cũng góp phần đáng kể vào thành tích học tập và rèn luyện của sinh viên [14].

\section{PHƯƠNG PHÁP NGHIÊN CÚU}

Nghiên cứu được tiến hành vào tháng 4/2018, tại trường Đại học Công nghiệp TPHCM (ĐHCN TPHCM). Nghiên cứu sử dụng phương pháp nghiên cứu định lượng. Dữ liệu được thu thập thông qua khảo sát bằng phiếu câu hỏi. Phương pháp chọn mẫu ngẫu nhiên theo tầng (theo năm học) và theo cụm dựa trên đơn vị lớp học được sử dụng để chọn mẫu khảo sát. Đầu tiên, dân số nghiên cứu được chia thành 2 nhóm theo năm học: nhóm thứ nhất bao gồm các sinh viên năm thứ nhất, nhóm thứ hai bao gồm các sinh viên năm thứ tư. Nhóm nghiên cứu chọn lựa khảo sát sinh viên năm thứ nhất và năm thứ tư do năm thứ nhất và năm thứ tư là hai cột mốc quan trọng nhất trong toàn bộ quá trình học tập ở bậc đại học. Năm thứ nhất đặt nền móng cho toàn bộ quá trình học tập, năm thứ tư là thời điểm kết thúc quá trình học tập. Từ 
danh sách các lớp học có trong từng nhóm, nhóm nghiên cứu sẽ chọn lựa ngẫu nhiên các lớp tham gia vào khảo sát.

Kích thước mẫu được xác định dựa trên công thức của Tabachnick và Fidell (2007):

$$
N=8^{*} \text { var }+50
$$

trong đó $\mathbf{N}$ là kích thước mẫu, var là số biến độc lập đưa vào mô hình hồi quy [15]. Mô hình hồi quy đa biến của nghiên cứu này có 10 biến độc lập, như vậy kích thước mẫu tối thiểu sẽ là $8 * 10+50=130$.

Dựa trên nguyên tắc: mẫu càng lớn, kết quả càng chính xác và dựa trên điều kiện kinh phí, thời gian nghiên cứu, nhóm nghiên cứu quyết định khảo sát từ 480 - 550 sinh viên. Nhóm đã chọn ngẫu nhiên 8 lớp năm thứ nhất và 10 lớp năm thứ tư. Nhóm liên hệ với giảng viên giảng dạy các lớp trên để xin phép được khảo sát. Mười lớp (4 lớp năm nhất và 6 lớp năm tư) đồng ý tham gia khảo sát đầu tiên đã được chọn làm mẫu khảo sát. Tổng số sinh viên của 10 lớp là 543 sinh viên. Tổng số phiếu khảo sát thu được sau khi tiến hành khảo sát là $500 / 543$. Trong số 500 sinh viên tham gia khảo sát, về giới tính, nam sinh viên chiếm $31 \%$ (155) và nữ chiếm 69\% (345). Về chuyên ngành học, mẫu khảo sát phân bố ở hầu hết các chuyên ngành đào tạo tại ĐHCN TPHCM. Trong nghiên cứu này, mẫu khảo sát được chia thành hai nhóm lớn: nhóm kỹ thuật (điện tử, công nghệ thông tin, cơ khí ...) và nhóm kinh tế (kế toán, quản trị kinh doanh, thương mại du lịch ...). Nhóm kỹ thuật chiếm 55,2\% (276), nhóm kinh tế chiếm 44,8\% (224). Về năm học, mẫu khảo sát có 250 sinh viên năm thứ nhất $(50 \%)$ và 250 sinh viên năm thứ tư $(50 \%)$.

\begin{tabular}{|c|c|c|c|}
\hline Đăc điểm & & Số lương & Tỷ lê $(\%)$ \\
\hline \multirow[t]{2}{*}{ Giới tính } & Nam & 155 & 31 \\
\hline & Nữ & 345 & 69 \\
\hline \multirow[t]{2}{*}{ Năm học } & Thứ nhất & 250 & 50 \\
\hline & Thứ tư & 250 & 50 \\
\hline \multirow[t]{2}{*}{ Ngành học } & Kỹ thuật & 276 & 55,2 \\
\hline & Kinh tế & 224 & 44,8 \\
\hline
\end{tabular}

Phiếu câu hỏi khảo sát sử dụng trong nghiên cứu được xây dựng dựa trên bảng câu hỏi College Survey Report (CSR). Bảng câu hỏi CSR đã được sử dụng trong các cuộc khảo sát cấp quốc gia hàng năm ở Mỹ về Student Engagement (National Survey of Student Engagement - NSSE) từ năm 2000 đến nay. CSR được xem là một trong những bảng câu hỏi khảo sát có độ tin cậy cao và có uy tín nhất về NLHT và HĐGS\&HT của nhà trường (hai khái niệm quan trọng không thể tách rời của Student Engagement). Trung Quốc, Úc, New Zealand, Nam Phi, Tây Ban Nha... đã mua lại bản quyền CSR để sử dụng trong các khảo sát tại các trường đại học ở các quốc gia này. Nội dung của phiên bản CSR 2018 có thể tìm hiểu tại [16]. Nhóm nghiên cứu đã thực hiện một số điều chỉnh (bỏ bớt câu hỏi, thêm câu hỏi, sửa nội dung một số câu hỏi) để phiếu khảo sát phù hợp hơn với điều kiện và môi trường giáo dục ở Việt Nam. Phiếu câu hỏi bao gồm 22 câu hỏi lớn, 98 mục hỏi. Các câu hỏi yêu cầu sinh viên đánh giá các hoạt động học tập sinh viên thực hiện tại trường, các hoạt động giảng dạy, hỗ trợ của nhà trường, các kết quả học tập và phát triển nhân cách mà sinh viên đạt được trong thời gian theo học tại trường. Thang đo Likert (4 điểm, 5 điểm, 7 điểm và 8 điểm) được sử dụng để đo lường các đánh giá của sinh viên.

Nhóm nghiên cứu xây dựng thang đo NLHT dựa trên tài liệu và các nghiên cứu trước đó ở Mỹ và ở Úc về NLHT $[4,5]$, thang đo HĐGD\&HT được xây dựng dựa trên thang đo của khảo sát NSSE của Mỹ. Ngoại trừ nhân tố Thách thức thi cử trong thang đo HĐGD\&HT chỉ chứa 1 mục hỏi, nhóm nghiên cứu sử dụng hệ số tương quan biến tổng và hệ số Cronbach's alpha để đánh giá độ tin cậy của các nhân tố trong 2 thang đo. Đa số các biến quan sát được chọn trong các nhân tố HĐGD\&HT có hệ số tương quan biến tổng > 0,40. Chỉ có 3 biến quan sát có hệ số tương quan biến tổng là $0,29,0,31$ và 0,34 . Sau khi xem xét giá trị Cronbach's alpha nếu loại bỏ biến và đặc biệt, giá trị nội dung của các biến này đối với nhân tố, nhóm quyết định vẫn giữ lại 3 biến trên. Riêng với thang đo NLHT, ban đầu nhóm nghiên cứu chọn lựa 7 mục hỏi. Nhưng sau khi kiểm tra hệ số tương quan biển tổng, nhóm quyết định loại bỏ 2 biến có hệ số tương quan biến tổng là 0,22 và 0,19 . Thang đo NLHT chỉ còn chứa 5 biến quan sát. Tổng cộng có 44 mục hỏi đã được dùng để xây dựng 2 thang đo. Tên các thang đo, số mục hỏi, nội dung của thang đo được trình bày chi tiết trong bảng 2 . 
TRƯỜNG ĐẠI HỌC CÔNG NGHIỆP TPHCM

Bảng 2: Các thang đo NLHT của sinh viên và các HĐGD\&HT của nhà truò̀ng

\begin{tabular}{|c|c|c|c|}
\hline Thang do & Nhân tố & Số mục hỏi & Dũ̃ liệu thu thập \\
\hline $\begin{array}{l}\text { Nỗ lực học } \\
\text { tập của } \\
\text { sinh viên } \\
\text { (NLHT) }\end{array}$ & $\begin{array}{l}\text { Nỗ lực học tập của sinh } \\
\text { viên }\end{array}$ & 5 & $\begin{array}{l}\text { Thời gian sinh viên dành cho việc chuẩn bị bài, } \\
\text { Mức độ sinh viên thường xuyên: chuấn bị bài trước khi } \\
\text { đến lớp; cố gắng nắm vững các nội dung khó trong bài, } \\
\text { hoàn thiện ở mức tốt nhất các bài tập, tiểu luận; cố gắng } \\
\text { hết sức để có thể đáp ứng được các yêu cầu, tiêu chuẩn } \\
\text { của giảng viên. }\end{array}$ \\
\hline \multirow{10}{*}{ 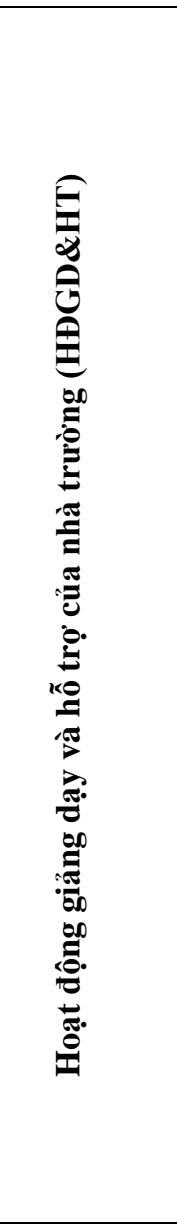 } & $\begin{array}{l}\text { Phát triển tư duy bậc } \\
\text { cao }\end{array}$ & 4 & $\begin{array}{l}\text { Mức độ chú trọng của chương trình đào tạo đến việc phát } \\
\text { triển các hoạt động tư duy bậc cao: phân tích, đánh giá, } \\
\text { vận dụng kiển thức và tạo ra kiến thức mới. }\end{array}$ \\
\hline & $\begin{array}{l}\text { Học tập thông qua tích } \\
\text { hợp và chiêm nghiệm }\end{array}$ & 6 & $\begin{array}{l}\text { Mức độ sinh viên thường xuyên tích hợp kiến thức từ } \\
\text { nhiều nguồn tài liệu, môn học khác nhau trong các bài } \\
\text { tiểu luận, kiểm tra; liên kết kiến thức được hợc với kinh } \\
\text { nghiệm bản thân; phân tích điểm mạnh, yếu trong lập } \\
\text { luận của bản thân, hiểu quan điểm của người khác ... }\end{array}$ \\
\hline & Chiên lược học tập & 3 & $\begin{array}{l}\text { Mức độ sinh viên thường xuyên xác định thông tin } \\
\text { chính, xem lại ghi chép, tóm tắt, tổng kết bài học, tài liệu }\end{array}$ \\
\hline & Lập luận định lượng & 3 & $\begin{array}{l}\text { Mức độ sinh viên thường xuyên sử dụng số liệu để đưa } \\
\text { ra kết luận, phân tích vấn đề thực tế và đánh giá kết luận } \\
\text { của người khác. }\end{array}$ \\
\hline & Hợp tác trong học tập & 4 & $\begin{array}{l}\text { Mức độ sinh viên thường xuyên hợp tác với bạn học khi } \\
\text { tìm hiểu tài liệu, làm đồ án, tiểu luận, ôn thi. }\end{array}$ \\
\hline & $\begin{array}{l}\text { Tương tác giữa sinh } \\
\text { viên và giảng viên }\end{array}$ & 5 & $\begin{array}{l}\text { Mức độ sinh viên thường xuyên trao đổi với giảng viên } \\
\text { về bài học trong và ngoăi lớp học, về kết quả học tập, về } \\
\text { dự định nghề nghiệp và cùng tham gia với giảng viên } \\
\text { trong các hoạt động ngoại khóa. }\end{array}$ \\
\hline & $\begin{array}{l}\text { Hoạt động giảng dạy } \\
\text { hiệu quả }\end{array}$ & 5 & $\begin{array}{l}\text { Mức độ giảng viên thực hiện các hoạt động giảng dạy } \\
\text { sau: trình bày bài giảng logic, giải thích mục tiêu, yêu } \\
\text { cầu của môn học, sữ dụng ví dư, minh hoa bài giảng, } \\
\text { nhận xét, phản hồi bài tập, bài kiềm tra của sinh viên...). }\end{array}$ \\
\hline & Chất lượng tương tác & 4 & $\begin{array}{l}\text { Sinh viên đánh giá chất lượng mối quan hệ của mình với } \\
\text { bạn học, với giảng viên, cố vấn học tập và nhân viên } \\
\text { phòng ban. }\end{array}$ \\
\hline & $\begin{array}{l}\text { Sự hỗ trợ của nhà } \\
\text { trường }\end{array}$ & 4 & $\begin{array}{l}\text { Mức độ chú trọng của nhà trường đối với các hoạt động } \\
\text { hỗ trợ sinh viên trong học tập và trong cuộc sống. }\end{array}$ \\
\hline & Thách thức thi cử & 1 & Mức độ các kỳ thi buộc sinh viên phải học hết sức mình \\
\hline
\end{tabular}

Kết quả kiểm định Cronbach's alpha cho thấy, trừ Chiến lược học tập có hệ số Cronbach's alpha 0,65, ở các nhân tố còn lại hệ số này dao động từ 0,70 cho đến 0,85 . Hệ số tương quan biến tổng và hệ số Cronbach's alpha chứng tỏ các nhân tố trong 2 thang đo được sử dụng trong nghiên cứu này đã đạt được độ tin cậy cần thiết. Kết quả kiểm định Cronbach's alpha được trình bày trong bảng 3 .

Bảng 3: Hệ số Cronbach's alpha của các thang đo

\begin{tabular}{lcccccccccc}
\hline & TDB & TH\& & CLH & LLĐ & HTc & TTG & GDH & CLT & HTr & NLHT \\
& C & CN & T & L & & V-SV & Q & T & & \\
\hline Cronbach' & 0,76 & 0,77 & 0,65 & 0,76 & 0,70 & 0,80 & 0,85 & 0,84 & 0,77 & 0,70
\end{tabular}

s alpha $\alpha$

Chú thích: $T D B C=$ Phát triển tu duy bậc cao; TH\&CN=Học tập thông qua tích hợp và chiêm nghiệm, CLHT $=$ Chiến luợc hoc tập; $L L Đ L=$ Lập luận định luợng; HT = Họp tác trong học tập; TTGV-SV = Tương tác giữa sinh viên và giảng viên; $G D H Q=$ Hoạt động giảng dạy hiệu quả; CLTT = Chất luợng tuoong tác; Htr = Sụ hố trợ của nhà trường; NLHT = Nỗ lục học tập của sinh viên 
Để xác định ảnh hưởng của các HĐGD\&HT của ĐHCN TPHCM đối với NLHT của sinh viên, nhóm nghiên cứu đã xây dựng mô hình nghiên cứu trong đó 10 nhân tố của thang đo HĐGD\&HT đóng vai trò như các biến số độc lập, thang đo NLHT đóng vai trò như biến số phụ thuộc. Các biến độc lập được đưa vào mô hình nghiên cứu là các HĐGD\&HT, theo xác định của các nghiên cứu trước đó, có ảnh hường tích cực đến NLHT. Mô hình nghiên cứu được minh họa ở hình 1.

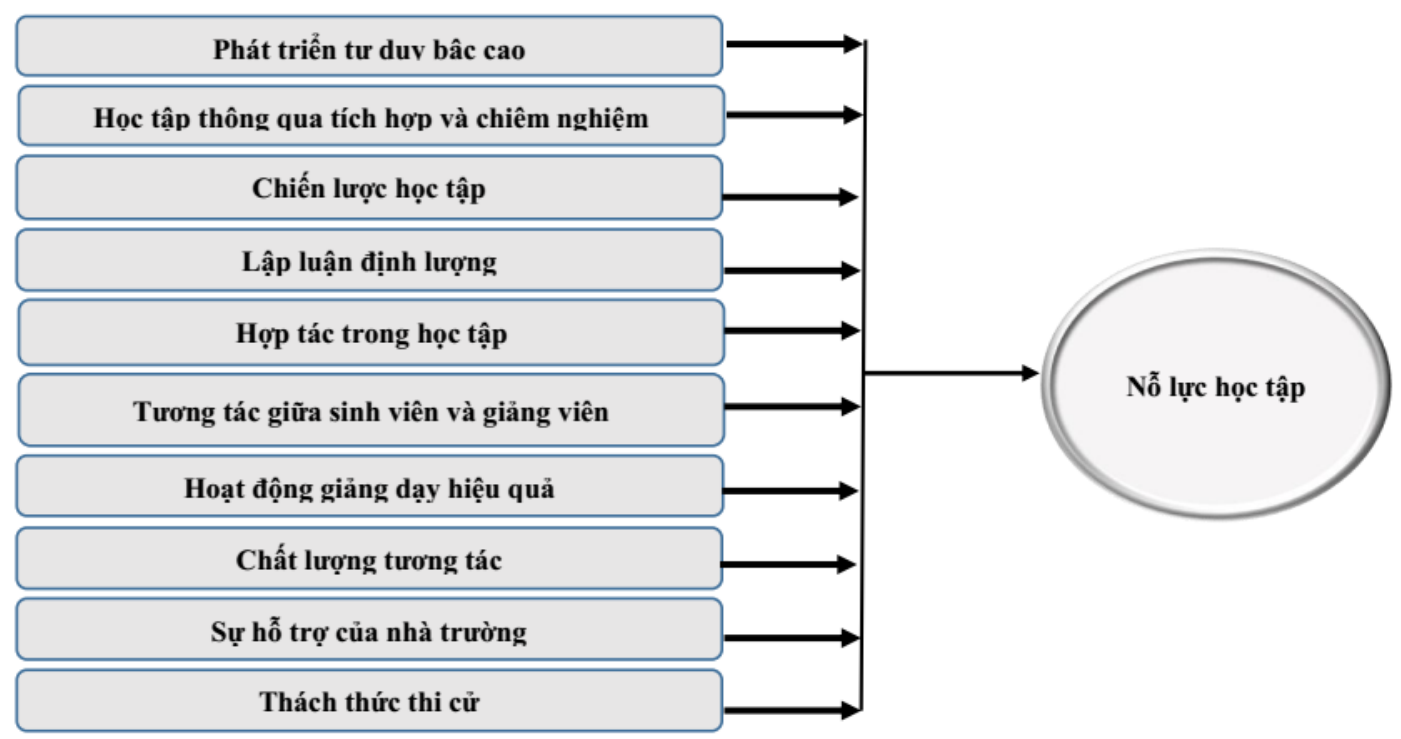

Hình 1: Mô hình nghiên cứu

Dữ liệu thu thập từ phiếu khảo sát được xử lý bằng phần mềm thống kê SPSS 22.0. Thống kê mô tả (giá trị trung bình, độ lệch chuẩn $(\mathrm{SD})$, tỷ lệ phần trăm) được sử dụng để phân tích các đặc điểm của mẫu khảo sát và mô tả các thang đo NLHT và các HĐGD\&HT. Để đánh giá ảnh hưởng của các HĐGD\&HT của ĐHCN TPHCM đối với NLHT của sinh viên, nhóm nghiên cứu sử dụng 2 phép tính thống kê: (i) phân tích tương quan để kiểm tra mối tương quan tuyến tính chặt chẽ giữa biến phụ thuộc với các biến độc lập, (ii) phân tích hồi quy tuyến tính bội để xác định ảnh hưởng của các biến độc lập lên biến phụ thuộc (NLHT). Kết quả của phân tích hồi quy sẽ giúp kiểm tra tổng thể mức độ tác động của các HĐGD\&HT cũng như mức độ đóng góp của từng HĐGD\&HT đối với NLHT của sinh viên. Dựa vào kết quả phân tích, nhóm nghiên cứu có thể xác định hoạt động nào đang có tác động tích cực đến NLHT của sinh viên và hoạt động nào cần cải thiện để nâng cao NLHT của sinh viên trong thời gian tới.

\section{KẾT QUẢ NGHIÊN CÚU VÀ THẢO LUẬN}

\section{1. Đặc điểm của các nhân tố trong thang đo NLHT và HĐGD\&HT}

Các nhân tố trong hai thang đo NLHT và HĐGD\&HT được tính trên thang điểm 60 vì các biến quan sát trong các nhân tố được đo lường bằng các thang đo tổng Likert với nhiều mức độ khác nhau. Việc đưa các nhân tố về cùng một thang điểm sẽ giúp việc tính giá trị trung bình của các nhân tố cũng như so sánh các nhân tố với nhau dễ dàng hơn.

Bảng 4: Trung bình (means) và độ lệch chuẩn (SD) của các nhân tố

\begin{tabular}{|c|c|c|c|c|c|c|c|c|c|c|c|}
\hline \multicolumn{11}{|c|}{ Các nhân tố } & \multirow[b]{2}{*}{$\begin{array}{l}\text { Nỗ lực } \\
\text { học tập }\end{array}$} \\
\hline Thang do & & & & Hoạ & dộng gi & ảng dạy & hỗ trọ & & & & \\
\hline Nhân tố & $\begin{array}{l}\text { TD } \\
\text { BC }\end{array}$ & $\begin{array}{l}\text { TH\&C } \\
\mathbf{N}\end{array}$ & $\begin{array}{l}\text { CL } \\
\text { HT }\end{array}$ & $\begin{array}{l}\text { LL } \\
\text { ĐL }\end{array}$ & HT & $\begin{array}{l}\text { TTGV- } \\
\text { SV }\end{array}$ & $\begin{array}{l}\text { GD } \\
\text { HQ }\end{array}$ & $\begin{array}{l}\text { CL } \\
\text { TT }\end{array}$ & HTr & $\begin{array}{l}\text { TT } \\
\text { TC }\end{array}$ & $\begin{array}{l}\text { NL } \\
\text { HT }\end{array}$ \\
\hline Trung bình & 28,63 & 30,81 & 33,40 & 24,13 & 33,60 & 18,11 & 37,99 & 32,81 & 36,03 & 39,68 & 31,67 \\
\hline $\begin{array}{l}\text { Độ lệch } \\
\text { chuẩn }\end{array}$ & 10,22 & 9,31 & 10,11 & 11,57 & 10,81 & 10,55 & 11,33 & 12,50 & 10,63 & 14,04 & 8,70 \\
\hline Số lượng & 497 & 496 & 497 & 496 & 499 & 499 & 497 & 495 & 499 & 497 & 499 \\
\hline
\end{tabular}


TRƯỜNG ĐẠI HỌC CÔNG NGHIỆP TPHCM

Chú thích: $T D B C=$ Phát triển tu duy bậc cao; TH\&CN=Học tập thông qua tích hợp và chiêm nghiệm, CLHT = Chiến luợc hoc tập; $L L Đ L=$ Lập luận định luợng; HT = Hợp tác trong học tậ; TTGV-SV = Tuơng tác giũa sinh viên và giảng viên; $G D H Q=$ Hoạt động giảng dạy hiệu quả; CLTT = Chất luợng tuoong tác; Htr = Sụ hố trợ của nhà truòng; TTTC = Thách thức thi cư; NLHT = Nố lục học tập của sinh viên.

Nhìn chung, các nhân tố trong thang đo NLHT và HĐGD\&HT chỉ ở mức trung bình thấp. Ngoại trừ nhân tố Thách thức thi cử $(\mathrm{M}=39,68)$; Hoạt động giảng dạy hiệu quả $(\mathrm{M}=37,99)$ và Sự hỗ trợ của nhà trường $(\mathrm{M}=36,03)$, giá trị trung bình của $5 / 11$ nhân tố chỉ đạt từ 30,81 đến 33,60 . Đáng chú ý, $3 / 11$ nhân tố (Phát triển tư duy bậc cao; Tương tác giữa giảng viên và sinh viên; Lập luận định lượng) có giá trị trung bình thấp hơn 30 . Khoảng cách giá trị trung bình cao nhất $(\mathrm{M}=39,68)$ và giá trị trung bình thấp nhất $(\mathrm{M}=18,11)$ khá rộng, lên đến 21,57.

Kết quả cho thấy mức độ tương tác giữa sinh viên và giảng viên còn thấp, thách thức học thuật ĐHCN TPHCM đặt ra cho sinh viên cũng chưa cao. Các nhân tố liên quan đến thách thức học thuật như Phát triển tư duy bậc cao; Học tập thông qua tích hợp và chiêm nghiệm; Lập luận định lượng đều có giá trị trung bình tương đối thấp. Dữ liệu cũng chỉ ra sinh viên chưa nỗ lực nhiều trong học tập. Nhân tố Thách thức thi cử đạt giá trị trung bình cao nhất. Điều này cho thấy đối với nhiều sinh viên, thi cử vẫn là một trong những nhân tố có tác động lớn đến nỗ lực học tập của họ. Từ dữ liệu cũng có thể nhìn thấy một số điểm tích cực như sinh viên đánh giá cao hoạt động giảng dạy của giảng viên và các hoạt động hỗ trợ của Nhà trường cũng như sinh viên thường xuyên hợp tác với bạn học trong học tập. Kết quả phân tích chi tiết các nhân tố trong các thang đo này có thể tham khảo thêm ở [17].

\section{2. Ảnh hưởng của các hoạt động giảng dạy, hỗ trọ̣ của ĐHCN TPHCM đối với nỗ lực học tập của} sinh viên

Để kiểm tra mối quan hệ giữa các HĐGD\&HT của ĐHCN TPHCM và NLHT, nhóm nghiên cứu sử dụng 2 phép tính thống kê: phân tích tương quan và phân tích hồi quy tuyến tính bội.

Phân tích tương quan giúp xác định mối quan hệ tương quan và mức độ tương quan giữa 10 biến độc lập của thang đo HĐGD\&HT và biến phụ thuộc NLHT. Các nhân tố được sẳp xếp theo giá trị giảm dần của hệ số tương quan Pearson.

Bảng 5: Tương quan giũa các HĐGD\&HT của ĐHCN TPHCM và NLHT

\begin{tabular}{lc}
\hline Hoạt động giảng dạy, hỗ trọ của ĐHCN TPHCM & Nỗ lụ̣c học tập của sinh viên \\
\hline Chiến lược học tập & $0,44^{* *}$ \\
Tương tác giữa sinh viên và giảng viên & $0,40^{* *}$ \\
Hợp tác trong học tập & $0,39^{* *}$ \\
Học tập thông qua tích hợp và chiêm nghiệm & $0,38^{* *}$ \\
Chất lượng tương tác & $0,37^{* *}$ \\
Phát triển tư duy bậc cao & $0,34 * *$ \\
Áp lực thi cử & $0,32^{* *}$ \\
Hoạt động giảng dạy hiệu quả & $0,31^{* *}$ \\
Lập luận định lượng & $0,26 * *$ \\
Sự hỗ trợ của nhà trường & $0,20^{* *}$ \\
\hline
\end{tabular}

Chú thích $* *=\mathrm{p}<0,01$

Kết quả cho thấy tất cả các biến độc lập đều có mối quan hệ tương quan đồng biến với biến phụ thuộc. Điều này chỉ ra tất cả các HĐGD\&HT của ĐHCN TPHCM được đưa vào mô hình nghiên cứu đều có ảnh hưởng tích cực đến NLHT. Tuy nhiên, hệ số tương quan Pearson giữa tất cả biến số độc lập và biến phụ thuộc không cao. Hệ số tương quan ở 8/10 biến số độc lập chỉ ở mức trung bình, biến thiên từ $0,31<\mathrm{r}<0,44$. Chiến lược học tập $(\mathrm{r}=0,44)$ và Tương tác giữa giảng viên và sinh viên $(\mathrm{r}=0,40)$ có hệ số tương quan với Nỗ lực học tập cao nhất. Hai biến độc lập Lập luận định lượng $(\mathrm{r}=0,26)$ và Sự hỗ trợ của nhà trường $(\mathrm{r}=$ $0,20)$ có hệ số tương quan ở mức yếu.

Sau khi phân tích tương quan, nhóm nghiên cứu sử dụng phân tích hồi quy tuyến tính bội. Phân tích hồi quy tuyến tính bội sẽ giúp xác định (i) cường độ ảnh hưởng chung của 10 HĐGD\&HT đối với NLHT; (ii) cường độ ảnh hưởng của từng HĐGD\&HT đối với NLHT. Trong mô hình hồi quy tuyến tính bội, dữ liệu được nhập thành 2 khối (block). Khối 1 bao gồm các biến quan sát liên quan đển các đặc điểm riêng của 
sinh viên như giới tính, năm học, khối ngành học và điểm thi đầu vào đại học. Việc đưa khối 1 vào mô hình hồi quy giúp kiểm tra xem các đặc điểm riêng của sinh viên có ảnh hưởng gì đến nỗ lực học tập của sinh viên không. Khối 2 của mô hình bao gồm 10 nhân tố của thang đo HĐGD\&HT. Kết quả phân tích hồi quy tuyến tính bội được trình bày ở bảng 6 và bảng 7 .

Bảng 6: Tóm tắt mô hình phân tích hồi quy

\begin{tabular}{|c|c|c|c|c|c|c|c|c|c|c|}
\hline \multirow[b]{2}{*}{ Model } & \multirow[b]{2}{*}{$\mathrm{R}$} & \multirow[b]{2}{*}{$\mathrm{R}^{2}$} & \multirow[b]{2}{*}{$\begin{array}{c}\mathrm{R}^{2} \\
\text { điều chỉnh }\end{array}$} & \multirow{2}{*}{$\begin{array}{c}\text { Sai số chuẩn } \\
\text { của ước } \\
\text { lượng }\end{array}$} & \multicolumn{5}{|c|}{ Thống kê thay đổi } & \multirow[t]{2}{*}{$\begin{array}{l}\text { Durbin- } \\
\text { Watson }\end{array}$} \\
\hline & & & & & $\begin{array}{c}\mathrm{R}^{2} \\
\text { thay đồi }\end{array}$ & $\begin{array}{c}\mathrm{F} \\
\text { thay đổi }\end{array}$ & df1 & df2 & $\begin{array}{c}\text { Mức ý nghĩa } \\
F \text { thay đổi }\end{array}$ & \\
\hline$\overline{1}$ & 0,122 & 0,015 & 0,005 & 8,71036 & 0,015 & 1,574 & 4 & 416 & 0,180 & \\
\hline 2 & 0,628 & 0,394 & 0,373 & 6,91500 & 0,379 & 25,406 & 10 & 406 & 0,000 & 1,958 \\
\hline
\end{tabular}

Kết quả phân tích trong bảng 6 cho thấy các đặc điểm riêng của sinh viên chỉ có ảnh hưởng rất nhỏ đối với NLHT và không có ý nghĩa về mặt thống $\mathrm{kê}\left(\mathrm{R}^{2}\right.$ điều chỉnh $\left.=0,005, \mathrm{~F}(4,416)=1,57, \mathrm{p}=0,18\right)$. Xét tổng thể, các HĐGD\&HT của ĐHCN TPHCM có ảnh hưởng đến NLHT và có ý nghĩa về mặt thống kê $\left(\mathrm{R}^{2}\right.$ điều chỉnh $=0,37, \mathrm{~F}(10,406)=25,41, \mathrm{p}=0,000)$. Điều này phù hợp với nhận định của các nhà nghiên cứu trước đó $[10,18]$ về vai trò của các HĐGD\&HT của nhà trường trong việc thúc đẩy sinh viên học tập. Mười biến độc lập, 10 HĐGD\&HT, trong mô hình giải thích được khoảng $38 \%$ phương sai của biến phụ thuộc - NLHT $\left(\mathrm{R}^{2}\right.$ thay đổi $\left.=0,38\right)$.

Bảng 7: Kết quả phân tích hồi quy về ảnh hương của các HĐGD\&HT đối với NLHT

\begin{tabular}{|c|c|c|c|c|c|c|c|}
\hline \multirow[b]{2}{*}{ Nhân tố } & \multicolumn{2}{|c|}{$\begin{array}{c}\text { Hệ số } \\
\text { chưa chuẩn hóa }\end{array}$} & \multirow{2}{*}{$\begin{array}{c}\text { Hệ số } \\
\text { chuẩn hóa } \\
\text { Beta }\end{array}$} & \multirow[b]{2}{*}{$\mathbf{t}$} & \multirow{2}{*}{$\begin{array}{c}\text { Mức ý } \\
\text { nghĩa (p) }\end{array}$} & \multicolumn{2}{|c|}{$\begin{array}{c}\text { Thống kê } \\
\text { đa cộng tuyến }\end{array}$} \\
\hline & B & Độ lệch chuẩn & & & & Tolerance & VIF \\
\hline (Hằng số) & 5,291 & 6,014 & & 0,880 & 0,379 & & \\
\hline Giới tính & 0,475 & 0,792 & 0,025 & 0,600 & 0,549 & 0,860 & 1,163 \\
\hline Điểm thi ĐH & 0,007 & 0,253 & 0,001 & 0,028 & 0,977 & 0,853 & 1,172 \\
\hline Năm học & 0,139 & 0,243 & 0,024 & 0,571 & 0,568 & 0,864 & 1,157 \\
\hline Ngành học & 0,095 & 0,717 & 0,005 & 0,133 & 0,894 & 0,892 & 1,121 \\
\hline TDBC & 0,079 & 0,046 & 0,089 & 1,714 & 0,087 & 0,549 & 1,821 \\
\hline $\mathrm{TH} \& \mathrm{CN}$ & 0,088 & 0,053 & 0,089 & 1,670 & 0,096 & 0,520 & 1,925 \\
\hline CLHT & 0,209 & $\mathbf{0 , 0 3 8}$ & 0,241 & 5,454 & $\mathbf{0 , 0 0 0}$ & 0,765 & $\mathbf{1 , 3 0 7}$ \\
\hline LLĐL & $-0,074$ & 0,036 & $-0,096$ & $-2,043$ & 0,058 & 0,681 & 1,469 \\
\hline HT & 0,085 & $\mathbf{0 , 0 3 9}$ & 0,103 & 2,194 & 0,029 & 0,683 & 1,463 \\
\hline GDHQ & 0,053 & 0,036 & 0,068 & 1,474 & 0,141 & 0,701 & 1,426 \\
\hline TTGV-SV & 0,173 & 0,041 & 0,206 & 4,247 & $\mathbf{0 , 0 0 0}$ & 0,635 & $\mathbf{1 , 5 7 4}$ \\
\hline CLTT & $\mathbf{0 , 0 7 3}$ & $\mathbf{0 , 0 3 2}$ & 0,103 & 2,273 & 0,024 & 0,728 & $\mathbf{1 , 3 7 3}$ \\
\hline $\mathrm{Htr}$ &,- 013 &, 035 &,- 016 &,- 366 & ,715 &, 824 & 1,213 \\
\hline TTTC & 0,121 & $\mathbf{0 , 0 2 7}$ & 0,184 & 4,512 & 0,000 & 0,896 & 1,116 \\
\hline
\end{tabular}

Chú thích: $T D B C=$ Phát triển tu duy bậc cao; $T H \& C N=$ Học tập thông qua tích hợp và chiêm nghiệm, CLHT = Chiến luợc hoc tập; $L L Đ L=$ Lập luận định lương; HT = Họp tác trong học tập; TTGV-SV = Tương tác giữa sinh viên và giảng viên; $G D H Q=$ Hoạt động giảng dạy hiệu quả; CLTT = Chất luợng tuơng tác; Htr = Sụ hố trọ̆ của nhà truòng; TTTC = Thách thức thi củ. Biến phụ thuộc: NLHT = Nỗ lục học tập của sinh viên.

Các nhân tố có ảnh hưởng đến NLHT đự̛̣c tô đậm.

Số liệu từ bảng 7 cho thấy mức độ đóng góp của từng nhân tố đối với NLHT của sinh viên. Dựa vào mức ý nghĩa (p) có thể đưa ra kết luận ban đầu như sau: (i) tất cả các nhân tố liên quan đến đặc điểm riêng của sinh viên đều không có ảnh hưởng đến NLHT và (ii) chỉ có 5/10 HĐGD\&HT của ĐHCN TPHCM có tác động tích cực đến NLHT. Kết quả trên có thể biểu diễn qua phương trình hồi quy tuyến tính sau:

$$
\text { NLHT }=0,241 * \text { CLHT + 0,206*TTGV-SV + 0,184*TTTC + 0,103*HT + 0,103*CLTT }
$$

Kết quả trên đã chỉ ra rằng Chiến lược học tập $(B e t a=0,241)$ là nhân tố có ảnh hưởng lớn nhất đến NLHT. Những hoạt động như xác định ý chính trong bài học, đọc lại ghi chép, tóm tắt ý chính trong bài luôn đòi hỏi sinh viên phải đầu tư nhiều thời gian và công sức. Mức ảnh hưởng cao thứ hai thuộc về Tương tác giữa 
giảng viên và sinh viên $($ Beta $=0,206)$. Đây là điều khá bất ngờ do các phân tích mô tả cho thấy tương tác giữa sinh viên và sinh viên ở ĐHCN TPHCM còn rất thấp. Tuy nhiên, điều này lại tương thích với các nhận định của các nhà nghiên cứu Mỹ $[8,18,19]$ về vai trò của sự tương tác giữa giảng viên và sinh viên đối với NLHT. Theo các nhà nghiên cứu này, tương tác với giảng viên sẽ giúp sinh viên học thêm được nhiều kiến thức, kinh nghiệm cũng như sẽ nhận được sự tư vấn, động viên từ giảng viên. Sự tư vấn, động viên của giảng viên sẽ giúp sinh viên xác định được mục tiêu học tập, giúp sinh viên hứng thú trong học tập, qua đó, sẽ thúc đầy sinh viên nỗ lực nhiều hơn trong học tập. Thách thức thi cử cũng có những ảnh hưởng nhât định đối với NLHT. Trong điều kiện khi kiểm tra, đánh giá môn học của nhà trường vẫn đặt nặng vào các kỳ thi, ảnh hưởng của Thách thức thi cử đối với NLHT là điều không ngoài dự đoán. Tuy nhiên, hệ số Beta cho thấy nhân tố này ảnh hưởng không cao lắm đến NLHT. Điều này có thể do độ khó của các kỳ thi chưa cao, chưa buộc sinh viên phải đầu tư nhiều thời gian và công sức. Hợp tác trong học tập $(B e t a=0,103)$ và Chất lượng tương tác (Beta $=0,103)$ có ảnh hưởng không lớn đến NLHT.

Trong mô hình hồi quy, 5 nhân tố còn lại đều không có ảnh hưởng đến NLHT của sinh viên. Trong các nhân tố này, có đến 3 nhân tố liên quan đến Thách thức học thuật là Phát triển tư duy bậc cao; Học tập thông qua chiêm nghiệm và tích hợp và Lập luận định lượng. Kết quả này trái ngược với nhận định của một số nhà nghiên cứu Mỹ và Úc $[8,10,18,19]$. Các nhà nghiên cứu này luôn xem thách thức học thuật là một trong những biện pháp hiệu quả để thúc đẩy sinh viên học tập nhằm đạt được thành tích học tập tốt. Thách thức học thuật sẽ buộc sinh viên phải làm việc nhiều hơn, tư duy nhiều hơn, vì vậy, sẽ phải đầu tư nhiều thời gian và công sức cho học tập. Điểm khác biệt này có thể là do mức độ thách thức học thuật ĐHCN TPHCM đặt ra cho sinh viên còn khá thấp khi so sánh với mức độ thách thức học thuật mà các trường đại học Mỹ đặt ra cho sinh viên của mình [17]. Số liệu (means, tỷ lệ \%) của các nhân tố Phát triển tư duy bậc cao; Học tập thông qua chiêm nghiệm và tích hợp; Lập luận định lượng trong nghiên cứu này có sự chênh lệch đáng kể với số liệu của các nhân tố tương ứng trong khảo sát NSSE 2018 [17].

Trong mô hình hồi quy này, 2 nhân tố khác: Hoạt động giảng dạy hiệu quả và Sự hỗ trợ của nhà trường cũng không có ảnh hưởng đến NLHT mặc dù kết quả phân tích thống kê mô tả cho thấy sinh viên đánh giá khá cao 2 nhân tố này. Kểt quả này cũng không phù hợp với các nhận định của nhà nghiên cứu phương Tây $[8,19]$ về tác động của 2 nhân tố này đối với NLHT. Đối với nhân tố Sự hỗ trợ của nhà trường, kết quả trên có thể giải thích qua mức độ sử dụng của sinh viên đối với các dịch vụ hỗ trợ mà ĐHCN TPHCM dành cho sinh viên. Trong nghiên cứu này, khi được hỏi ý kiến về một số dịch vụ hỗ trợ sinh viên như hỗ trợ nghề nghiệp, tư vấn nghề nghiệp, tư vấn học tập, thư viện, khá nhiều sinh viên cho biết họ không thường xuyên sử dụng các dịch vụ hỗ trợ này trong năm học. Ví dụ, có đến $44,1 \%$ sinh viên chưa từng sử dụng dịch vụ hỗ trợ nghề nghiệp, $24,2 \%$ sinh viên cho rằng họ chưa được tư vấn về học tập. Đặc biệt, có đến $19,2 \%$ sinh viên không sử dụng thư viện, $28,7 \%$ sinh viên chỉ sử dụng thư viện 1 lần trong năm học. Số sinh viên sử dụng thư viện trên 5 lần trong năm học chỉ chiếm $16 \%$. Về trường hợp nhân tổ Hoạt động giảng dạy hiệu quả, theo lý thuyết, cách truyền đạt logic, hiệu quả của giảng viên hay phản hồi của giảng viên sẽ giúp sinh viên tiếp thu tốt hơn, tạo hứng thú học tập, hay sẽ có tác dụng giám sát tiến độ học tập của sinh viên, qua đó, có thể thúc đẩy sinh viên nỗ lực học tập. Tuy nhiên, kết quả phân tích dữ liệu của khảo sát này lại cho thấy Hoạt động giảng dạy hiệu quả không có ảnh hưởng đối với NLHT của sinh viên. Cần có thêm nghiên cứu và thu thập thêm dữ liệu để giải thích tác động của hoạt động giảng dạy hiệu quả đối với NLHT của sinh viên trong môi trường giáo dục đại học Việt Nam.

Trong phiếu khảo sát, sinh viên còn được yêu cầu chọn lựa nhân tố có ảnh hưởng cao nhất đến NLHT của họ. Thống kê mô tả của câu hỏi này được trình bày ở bảng 8 . Trong số 6 nhân tố được đưa ra trong câu hỏi, nhân tố 'sự chú trọng phát triển các kỹ năng tư duy bậc cao' được nhiều sinh viên chọn lựa nhất $(155,31 \%)$. Kết quả này cho thấy bản thân sinh viên cũng nhận thức được vai trò của các hoạt động giảng dạy và học tập hướng đến việc phát triển tư duy bậc cao của sinh viên đối với nỗ lực học tập. Tuy nhiên, theo kết quả phân tích hồi quy ở trên, nhân tố Phát triển tư duy bậc cao không có ảnh hưởng đối với NLHT một phần do sự ít chú trọng đến nhân tố này trong chương trình đào tạo, trong kiểm tra, đánh giá của ĐHCN TPHCM. Để nâng cao NLHT, ĐHCN TPHCM cần tăng cường những hoạt động phát triển tư duy bậc cao cũng như những hoạt động có liên quan đến thách thức học thuật khác vào chương trình đào tạo. Trong chọn lựa của sinh viên, tiếp theo 'sự chú trọng phát triển các kỹ năng tư duy bậc cao' là các nhân tố 'độ khó của các bài kiểm tra đánh giá' $(111,22,3 \%)$ và 'sự khuyến khích, thúc đẩy sinh viên nỗ lực của giảng viên' (107, 21,5\%). Kết quả này đã hỗ trợ cho kết quả phân tích hồi quy ở trên về mức độ ảnh hưởng của Thách thức 
thi cử và Tương tác giữa giảng viên và sinh viên đối với Nỗ lực học tập. Ba nhân tố còn lại có tỷ lệ sinh viên chọn lựa thấp, không đáng kể.

Bảng 8: Ý kiến của sinh viên về các nhân tố có ảnh hương cao nhất đến NLHT

\begin{tabular}{lcc}
\hline Nhân tố & Số lượng & Tỷ lệ \% \\
\hline Sự chú trọng phát triển các kỹ năng tư duy bậc cao & 155 & 31.1 \\
Độ khó của các bài kiểm tra đánh giá & 111 & 22.3 \\
Sự khuyến khích, thúc đẩy sinh viên nỗ lực học tập của giảng viên & 107 & 21.5 \\
Sự hỗ trợ của nhà trường cho sinh viên trong học tập và sinh hoạt & 78 & 15.7 \\
Môi trường giao tiếp thân thiện giữa sinh viên với sinh viên, giữa sinh viên với & 41 & 8.2 \\
Các hoạt động ngoại khóa & 6 & 1.2 \\
\cline { 2 - 3 } Tổng cộng & 500 & 100 \\
\hline
\end{tabular}

\section{KẾT LUẬN VÀ KHUYẾN NGH!}

Nghiên cứu này đã khảo sát mối quan hệ tuyến tính giữa các HĐGD\&HT của trường ĐHCN TPHCM và NLHT qua 2 phép tính thống kê: phân tích tương quan và phân tích hồi quy tuyến tính bội. Mười nhân tố trong thang đo HĐGD\&HT đóng vai trò như các biến độc lập, NLHT đóng vai trò như biến phụ thuộc. Phân tích tương quan cho thấy tất cả 10 nhân tố đều có mối tương quan tích cực đến NLHT. Tuy nhiên, mức độ tương quan của $8 / 10$ nhân tố chỉ ở mức trung bình $(0,31<\mathrm{r}<0,44), 2 / 10$ nhân tố có hệ số tương quan ở mức yếu. Trong mô hình hồi quy tuyến tính, các đặc điểm riêng của sinh viên không có ảnh hưởng đến NLHT, trong khi đó xét tổng thể các HĐGD\&HT của ĐHCN TPHCM có ảnh hưởng tích cực đến NLHT. Tuy nhiên, mức độ ảnh hưởng của các HĐGD\&HT không cao, các HĐGD\&HT chỉ có thể giải thích được 38\% phương sai của NLHT. Mức độ ảnh hưởng thấp của các HĐGD\&HT có thể do các nguyên nhân sau:

- Chỉ có 5/10 nhân tố có ảnh hưởng đến NLHT. Các nhân tố bao gồm: Chiến lược học tập; Tương tác giữa giảng viên; Thách thức thi cử; Hợp tác trong học tập và Chất lượng tương tác (được sắp xếp theo mức ảnh hưởng giảm dần). Kết quả này phù hợp với các nhận định về ảnh hưởng của các nhân tố trên đối với NLHT trong các nghiên cứu trước đó. Một điều đáng chú ý là mặc dù sinh viên đánh giá mức độ tương tác giữa giảng viên và sinh viên khá thấp nhưng nhân tố này lại có mức ảnh hưởng đến NLHT của sinh viên đứng hàng thứ hai chỉ sau Chiến lược học tập. Do kiểm tra đánh giá của nhà trường chủ yếu đặt nặng vào các kỳ thi giữa kỳ và cuối kỳ (chiếm $80 \%$ trọng số điểm tổng kết môn học), Thách thức thi cử được dự đoán là nhân tố có ảnh hưởng lớn nhất lớn NLHT của sinh viên. Tuy nhiên, kết quả hồi quy cho thấy mức ảnh hưởng của nhân tố này chỉ xếp ở hàng thứ ba với hệ số Beta không cao. Điều này có thể do độ khó hay hình thức của các kỳ thi chưa đủ phù hợp để buộc sinh viên phải nỗ lực học tập.

- Trong số 5 nhân tố không có ảnh hưởng đến NLHT, có đến 3 nhân tố liên quan đến Thách thức học thuật là Phát triển tư duy bậc cao; Học tập thông qua tích hợp và chiêm nghiệm và Lập luận định lượng. Điều này có thể là do mức độ thách thức học thuật ĐHCN TPHCM đặt ra cho sinh viên chưa cao trong khi các nhà nghiên cứu Mỹ, Úc $[8,10,18,19]$ cho rằng thông qua thách thức học thuật các trường đại học có thể thúc đẩy sinh viên nỗ lực học tập ở mức cao nhất để có thể phát huy được tiềm năng của bản thân nhằm đạt được thành tích tốt nhất có thể.

- Hai nhân tố Hoạt động giảng dạy hiệu quả và Sự hỗ trợ của nhà trường được sinh viên đánh giá rất cao nhưng lại không có ảnh hưởng đến NLHT. Điều này cũng trái ngược với các nghiên cứu trước đó.

Từ kểt quả nghiên cứu trên, nhóm nghiên cứu đề xuất một số khuyến nghị để nâng cao NLHT. Theo đó, trường ĐHCN TPHCM cần:

1. Tạo điều kiện để nâng cao mức độ tương tác giữa giảng viên và sinh viên như cung cấp thời gian, không gian để sinh viên có thể gặp gỡ, trao đổi, xin ý kiến tư vấn từ giảng viên, khuyến khích giảng viên và sinh viên tương tác với nhau qua các hình thức trực tuyến, tạo điều kiện để sinh viên có thể tham gia các hoạt động nghiên cứu hay hoạt động ngoại khóa cùng giảng viên;

2. Đa dạng hóa các hình thức kiểm tra, đánh giá, không chỉ sử dụng thi cử như phương tiện chủ yếu để đánh giá sinh viên. Đồng thời, trường ĐHCN TPHCM cũng cần nâng thêm độ khó của các kỳ thi sao cho vừa phù hợp với trình độ của sinh viên vừa đặt ra cho sinh viên một số thách thức cần phải nỗ lực để vượt qua; 
3. Tăng cường các HĐGD\&HT hướng đến việc phát triển các kỹ năng tư duy bậc cao, khả năng tích hợp và chiêm nghiệm kiến thức, khả năng sử dụng lập luận định lượng cho sinh viên trong chương trình giảng dạy, trong kiểm tra, đánh giá;

4. Cải thiện thêm các nhân tố như Chiến lược học tập; Hợp tác trong học tập; Chất lượng tương tác để nâng mức ảnh hưởng của các nhân tố này đối với NLHT;

5. Cần tiến hành thêm một số nghiên cứu để tìm hiểu thêm một số điểm chưa giải thích được trong nghiên cứu này như vì sao các hoạt động giảng dạy hiệu quả của giảng viên chưa có ảnh hưởng đến NLHT. Bên cạnh đó, cần có thêm một số nghiên cứu định tính để tìm hiểu thêm bản chất, đặc điểm của NLHT, để xác định các HĐGD\&HT có ảnh hưởng đến NLHT trong điều kiện đặc thù của giáo dục đại học Việt Nam.

\section{LÒ̀I CẢM ƠN}

Nhóm tác giả chân thành cảm ơn Trường Đại học Công nghiệp TP.HCM đã cấp kinh phí để thực hiện đề tài nghiên cứu này theo Hợp đồng nghiên cứu khoa học số 18/HĐ-ĐHCN, mã số 183.CB01.

\section{TÀI LIỆU THAM KHẢO}

[1] Nguyen, T.T.T, 2016. Student engagement: A useful quality concept in the Vietnamese Higher Education (PhD Thesis).

[2] Nguyễn Thị Thu Trang \& Đỗ Văn Dũng, 2018. Student Engagement - a promising solution to quality improvement of Vietnamese higher education, Journal of Technical Education Science, Ho Chi Minh city University of Technology and Education, 47 (5), pp. 85 -91.

[3] Kuh, G.D., 2009. The National Survey of Student Engagement: Conceptual and empirical foundations, New Directions for Institutional Research, 5-20 (141), pp. 5-20.

[4] Zhao, C-M \& Kuh, GD 2004, 'Adding value: learning communities and student engagement', Research in Higher Education, 45 (2), pp. 115-138.

[5] Community College Survey of Student Engagement (CCSSE), 2018. 2018 Benchmark Frequency Distributions Main Survey, Center for Community College Student Engagement. Available at:

< http://www.ccsse.org/survey/reports/2018/standard_reports/CCSSE_2018_coh_freqs_stueff_std.pdf>

[6] Lê Văn Lập, 2011. Phương pháp giảng dạy và tâm lý giáo dục trong trường học- Kỹ năng quản lý và giảng dạy đạt hiệu quả cao, NXB Lao động.

[7] Hoàng Thị Thu Hiền \& Nguyễn Thị Lan, 2012 Giáo trình Tâm lý học, TPHCM: NXB Đại học Quốc gia TP.HCM.

[8] Đoàn Huy Oánh, 2005. Tâm lý sư phạm, TP.HCM: NXB Đại học Quốc gia TP.HCM.

[9] Nguyễn Công Khanh, 2008. Nghiên cứu phong cách học của sinh viên, Tạp chí Giáo dục, 202, tr. 7-10.

[10] Kuh, G.D., Kinzie, J., Buckley, J.A., Bridges, B.K. \& Hayek, J.C., 2007. Piecing together the student success puzzle: research, propositions, and recommendations, ASHE Higher Education Report, 32 (5), pp. 1-182.

[11] National Survey of Student Engagement (NSSE), 2013. Engagement indicators, NSSE, IN: Indiana University Center for Postsecondary Research, Bloomington,

Available at $\langle\mathrm{http}: / /$ nsse.indiana.edu/html/engagement_indicators.cfm $>$.

[12] Krause, K-L. \& Coates, H., 2008. Students' engagement in first-year university, Assessment \& Evaluation in Higher Education, 33 (5), pp. 493-505.

[13] Radloff, A. \& Coates, H., 2010. Doing more for learning: enhancing engagement and outcomes. Australasian Student Engagement Report, Camberwell: Australian Council for Educational Research (ACER. Available at: $<$ http://research.acer.edu.au/ausse/12/>

[14] Coates, H., 2008. Beyond happiness: managing engagement to enhance satisfaction and grades, Camberwell: Australian Council for Educational Research (ACER).

[15] Tabachnick, B.G \& Fidell, L.S, 2007, Using multivariate statistics, 5th edn, Pearson/Allyn \&amp; Bacon, Boston. 
[16] National Survey of Student Engagement (NSSE), 2018. Survey Instrument, NSSE, IN: Indiana University Center for Postsecondary Research, Bloomington,

Available at: < http://nsse.indiana.edu/pdf/survey_instruments/2018/NSSE18_Screenshot_US_English.pdf>.

[17] Nguyễn Thị Thu Trang, Ngô Ngọc Hưng \& Trần Anh Dũng, đang chờ xuất bản. Nỗ lực học tập của sinh viên và các hoạt động giảng dạy, hỗ trợ sinh viên tại trường Đại học Công nghiệp TP.HCM, Tạp chí Khoa học \& Công nghệ trường Đại học Công nghiệp TPHCM.

[18] Kuh, G.D., Kinzie, J., Schuh, J.H., Whitt, E.J. \& Associates, 2005. Student success in college: Creating conditions that matter, 1st edn, San Francisco: Jossey-Bass.

[19] Pascarella, ET \& Terenzini, P.T., 2005. How college affects students : a third decade of research, 2nd edn, San Francisco: Jossey-Bass.

Ngày nhận bài: 08/03/2019

Ngày chấp nhận đăng:03/04/2019 